\title{
Selectivity of Direct Ethanol Fuel Cell Dictated by Unique Partial Oxidation Channel
}

\author{
Hui-Fang Wang, Zhi-Pan Liu*
}

Shanghai Key laboratory of molecular catalysis and innovative materials, Department of Chemistry, Fudan University

Shanghai 200433 China

\section{Calculation details:}

The exchange-correlation functional used for the plane-wave DFT was GGA-PBE. Unless specifically mentioning, the slab used is four-layer $p(2 \times 2) \operatorname{Pt}(111)$, equivalent to $1 / 4 \mathrm{ML}$, with the top two-layer relaxed. This coverage is large enough to avoid the direct bonding sharing between adjacent image molecules; the convergence on the barrier has been verified at $p(3 \times 3)$ unit cell. A cutoff energy of $340 \mathrm{eV}$ was used. The convergence of the barrier was examined by increasing cutoff energy to $400 \mathrm{eV}$, which showed very small difference in the barrier (within $0.02 \mathrm{eV}$ ) and no change in relative activity between reactions that are critical to selectivity. Monkhorst-Pack k-point sampling with approximately $0.04 \times 2 \pi \AA^{-1}$ spacing in reciprocal lattice was utilized for all of the calculations. Transition states (TSs) of reaction was searched using the constrained minimization method and was then verified using vibrational frequency analysis (only one negative mode is present at the TS). The vibrational 
frequency analysis was carried out numerically using the finite displacement method, as utilized in our previous publications (Ref. 13 in letter).

In calculating the relative potential energy of an adsorbed state, the total energy of adsorbed $\mathrm{H}$ atom (1/4 ML) and adsorbed OH group (1/4 ML) and the adsorbed state (1/4 ML) are calculated independently (see equation 1 in manuscript), which mimics the adsorption of these species at low coverage conditions. This approach is routinely used in modeling surface reactions with periodic boundary condition (see for example ref. 16)

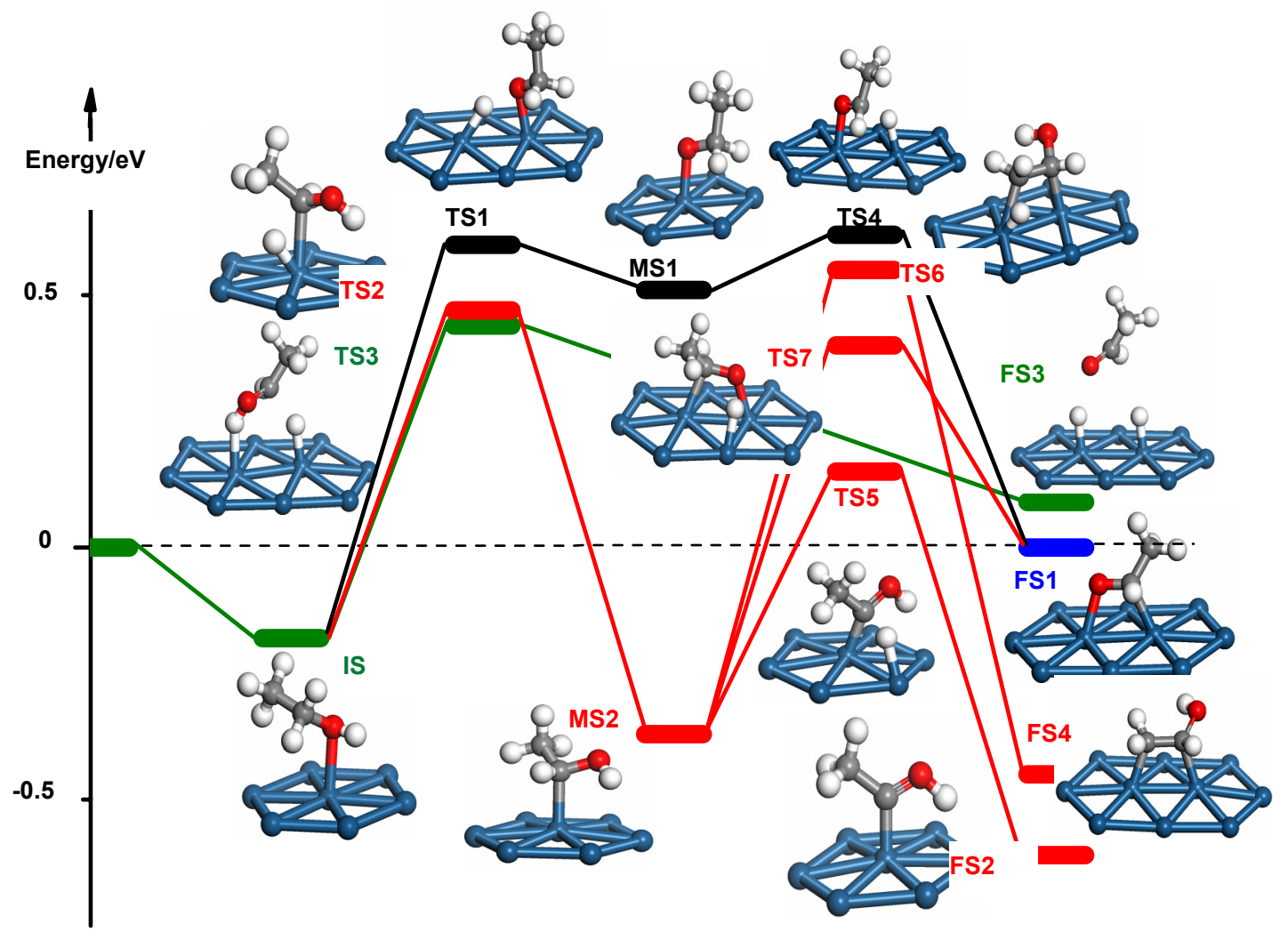

Figure S1: Complete potential energy diagram of ethanol oxidation leading to acetaldehyde (two additional pathways are shown compared to Figure 1 in the manuscript). IS: $\mathrm{CH} 3 \mathrm{CH} 2 \mathrm{OH}$ -0.18; TS1: 0.60; TS2: 0.47; TS3: 0.44; MS1: CH3CH2O 0.51; MS2: CH3CHOH -0.37; TS4: 0.62; TS5: 0.15; TS6: 0.55; TS7: 0.40; FS1: CH3CHO 0.0; FS2: CH3COH -0.61; FS3: CH3CHO* 0.09; FS4: $\mathrm{CH} 2 \mathrm{CHOH}-0.45$. 
Table S1: The enlarged view and the key structural parameters of the calculated intermediate states (also see S-Figure 1 and Figure 2 for the states). The unit of distance is $\AA$. Distances longer than $3 \AA$ are not listed.

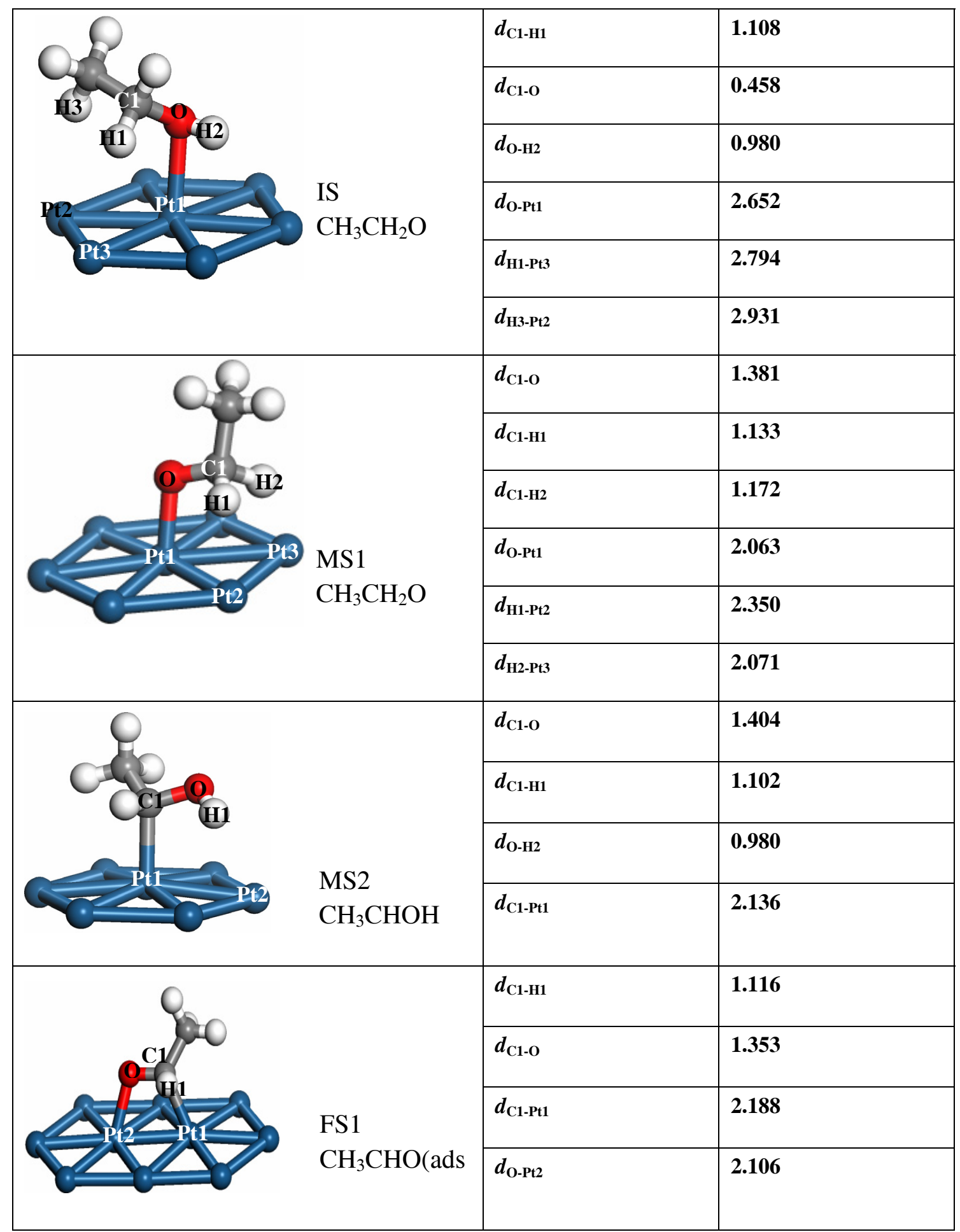




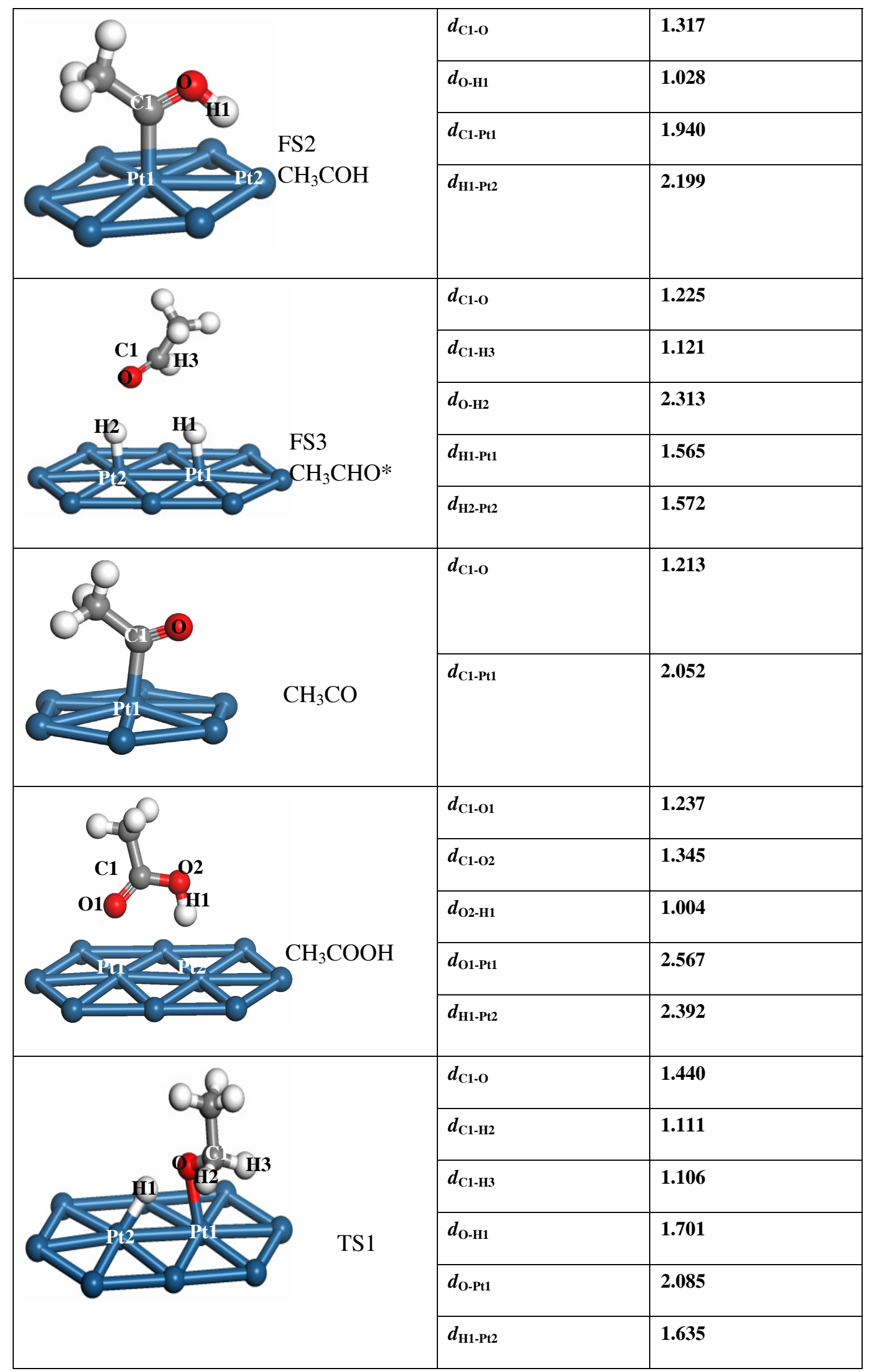




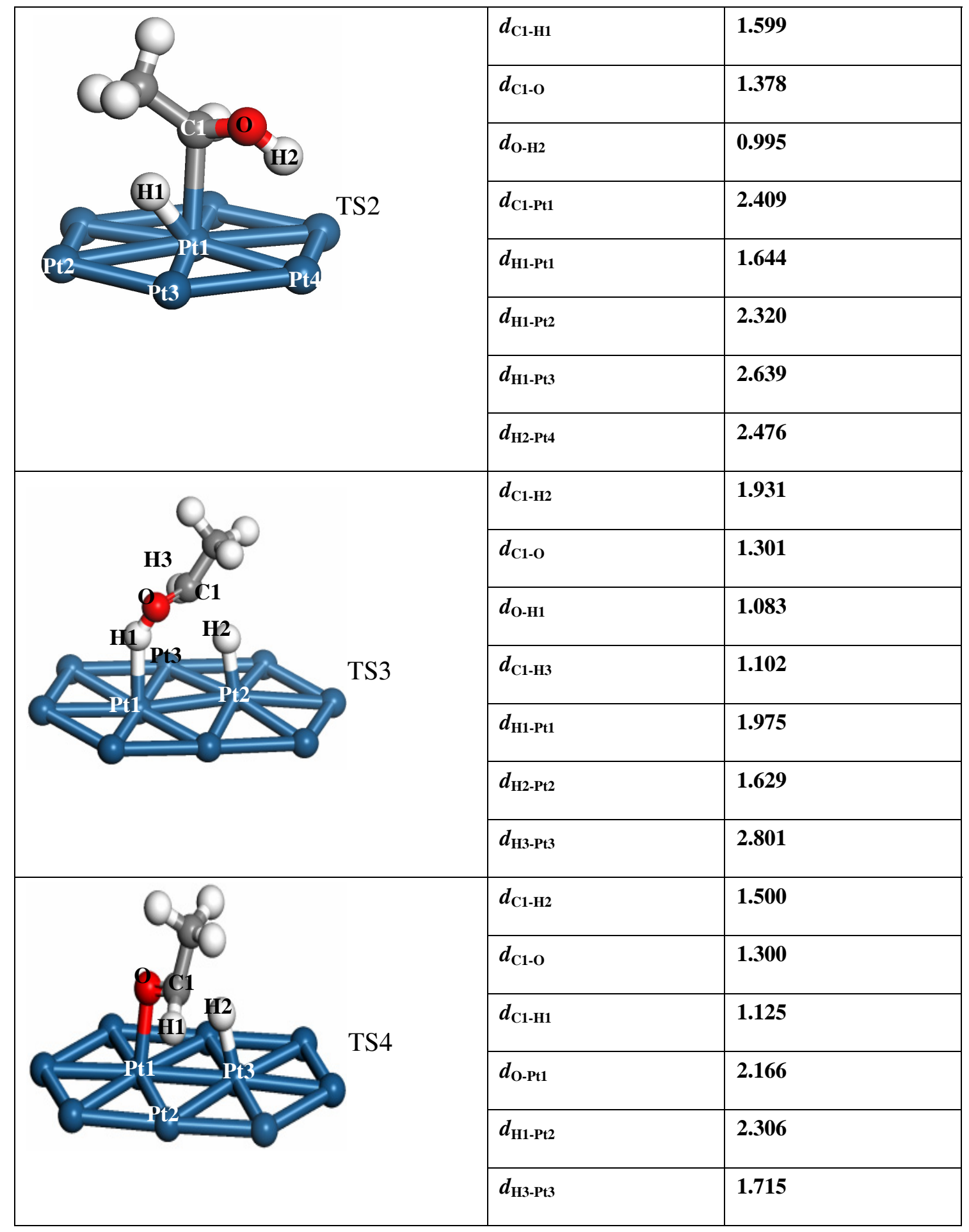




\begin{tabular}{|c|c|c|}
\hline \multirow{6}{*}{ TS5 } & $d_{\text {C1-O }}$ & 1.351 \\
\hline & $d_{\mathrm{C} 1-\mathrm{H} 1}$ & 1.535 \\
\hline & $d_{\mathrm{O}-\mathrm{H} 2}$ & 1.006 \\
\hline & $d_{\mathrm{C} 1-\mathrm{Pt1}}$ & 2.029 \\
\hline & $d_{\mathrm{H} 1-\mathrm{P} 2}$ & 1.684 \\
\hline & $d_{\mathrm{H} 2-\mathrm{Pt3}}$ & 2.313 \\
\hline \multirow[b]{5}{*}{ TS: $\mathrm{CH} 3 \mathrm{COH} \rightarrow \mathrm{CH} 3 \mathrm{CO}+\mathrm{H}$} & $d_{\mathrm{Cl}-\mathrm{O}}$ & 1.250 \\
\hline & $d_{\mathrm{O}-\mathrm{H} 1}$ & 1.455 \\
\hline & $d_{\mathrm{C} 1-\mathrm{Pt1}}$ & 2.004 \\
\hline & $d_{\mathrm{H} 1-\mathrm{P} 2}$ & 1.653 \\
\hline & & \\
\hline \multirow{5}{*}{$\mathrm{TS}: \mathrm{CH} 3 \mathrm{CHO} \rightarrow \mathrm{CH} 3 \mathrm{CO}+\mathrm{H}$} & $d_{\mathrm{C} 1-\mathrm{O}}$ & 1.308 \\
\hline & $d_{\mathrm{C} 1-\mathrm{H} 1}$ & 1.280 \\
\hline & $d_{\mathrm{C} 1-\mathrm{Pt} 1}$ & 2.140 \\
\hline & $d_{\mathrm{O}-\mathrm{Pt} 2}$ & 2.173 \\
\hline & $d_{\mathrm{H} 1-\mathrm{Pt3}}$ & 1.822 \\
\hline \multirow{6}{*}{$\mathrm{TS}: \mathrm{CH} 3 \mathrm{CO}+\mathrm{OH} \rightarrow \mathrm{CH} 3 \mathrm{COOH}$} & $d_{\mathrm{C1}-01}$ & 1.880 \\
\hline & $d_{\mathrm{C1}-\mathrm{O2} 2}$ & 1.225 \\
\hline & $d_{\text {C1-Pt1 }}$ & 2.175 \\
\hline & $d_{\text {O1-P12 }}$ & 2.127 \\
\hline & $d_{\text {O1-H1 }}$ & 0.984 \\
\hline & & \\
\hline
\end{tabular}




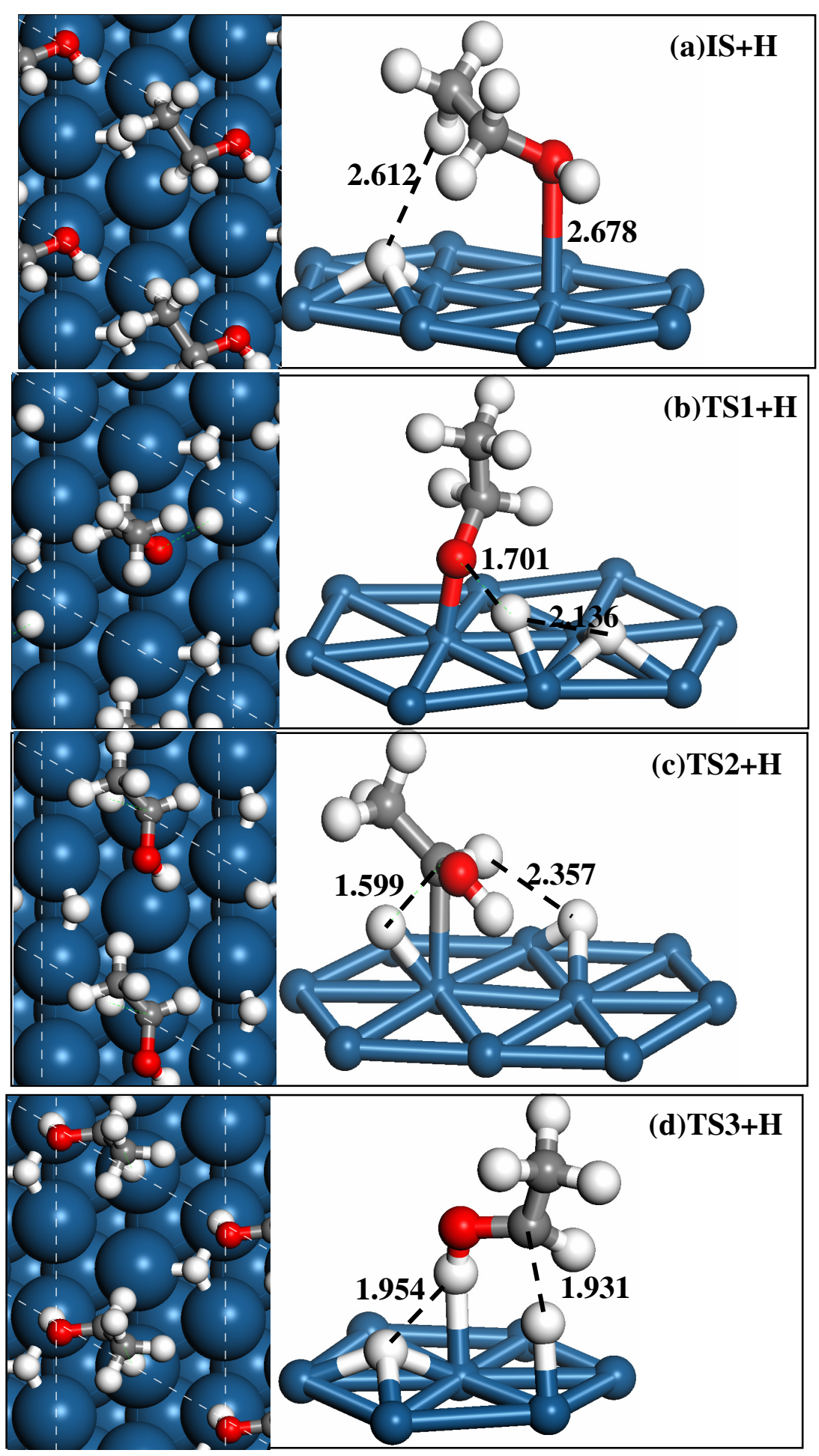

Figure S2. The top view (left) and enlarged side view (right) of the coadsorption systems with $\mathrm{H}$. The key distances $(\AA)$ are labeled. 

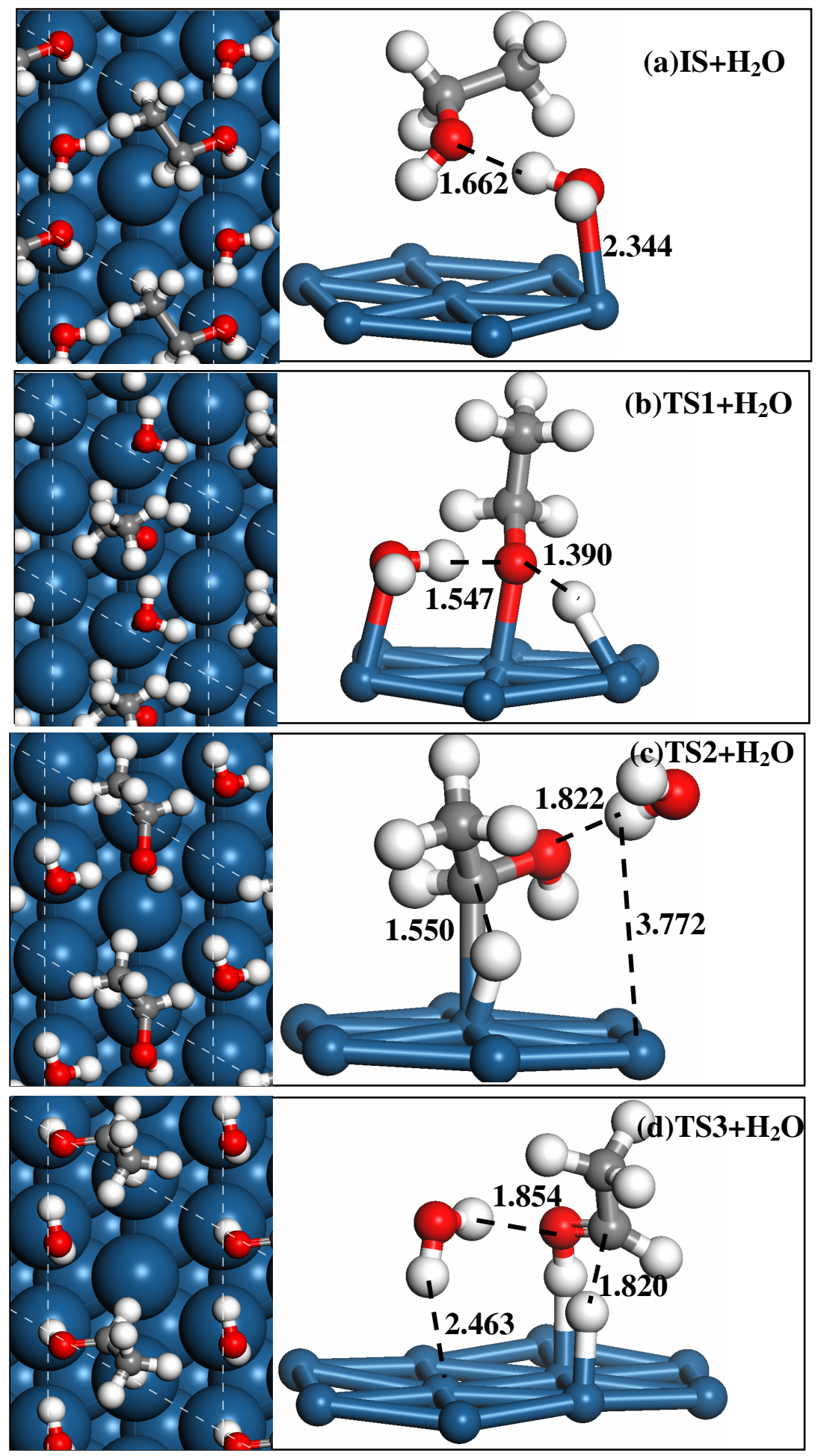

Figure S3. The top view (left) and enlarged side view (right) of the coadsorption systems with $\mathrm{H}_{2} \mathrm{O}$. The key distances $(\AA)$ are labeled. 